\title{
Design of Affinity Membrane Bioseparations
}

\author{
P. Sridhar*
}

Affinity separations rely on the highly specific binding between a protein in solution and an immobilized ligand to achieve a high degree of protein purification. A mathematical model including convection, diffusion, and rate kinetics is formulated to analyze the design and operation of affinity membrane bioseparations. The model equations are solved by orthogonal collocation method. Danckwerts' boundary conditions are used. The results obtained from model simulation show that the breakthrough of the protein is significantly influenced by Péclet number, feed protein concentration, Ligand number, Damköhler number, membrane thickness, and flow rate. Breakthrough profiles are quantitatively discussed in terms of protein recovery efficiency, ligand utilization efficiency, thickness of unused membrane, and width of the mass transfer zone.

\section{Introduction}

Selective binding capabilities offer advantages over relatively non-specific physical properties when used as basis of separation process. Affinity separations utilize the highly specific binding between a protein in solution and an immobilized ligand to attain a high level of protein purity. Conventionally, affinity separation protocols require pumping the protein solution through a packed bed containing porous beads onto which the ligand is immobilized. A number of interesting developments have emerged over the past few years. These include continuous extraction [1], affinity cross-flow filtration [2], radial-flow [3], and magnetically fluidized columns [4], affinity expanded beds [5], and perfusion chromatography [6].

An affinity device using mircoporous membranes as support matrix with sites on the pore wall for ligand attachment offers high surface area, reduces the diffusion distance, and requires low operating pressure $[7,8]$. These will result in high-volume throughout, high efficiency of ligand utilization, and low cost. Affinity membranes are a new alternative to packed beds, and have the unique advantage that the separation rate frequently approaches the fundamental rate limit set by the kinetics of ligand solute association and dissociation [9]. This is not true in packed beds, where the separation rate is limited by either slow intrabead diffusion for large beads or low axial velocities and high column pressure drops for small beads [10]. These limitations result in long times for the steps of adsorption, washing, and elution, which is uneconomical. Pressure drop and intra-bead diffusion limitations are minimized by convection through the fine pores (pore size $<1 \mu \mathrm{m}$ ) of the membrane [11]. Axial diffusion, which is not significant in long packed beds [12], may become a dominant factor in these thin membranes. However, the proper design and operation of affinity membrane separations require a fundamental understanding of the underlying transport phenomena and adsorption equilibrium behavior.

\footnotetext{
* P. Sridhar, Research Associate, Department of Chemical Engineering, Indian Institute of Science, Bangalore 560012 , India.
}

Process modeling and simulation are conducted to gain a better understanding of the solute breakthrough behavior in an affinity membrane. The objective of the present study is to develop a mathematical model that describes the adsorption stage of affinity membrane from which the breakthrough curves can be generated. This comprehensive model includes axial diffusion and surface reaction kinetics. The solution of this comprehensive model requires numerical computation. Orthogonal collocation method [13-14] is used to evaluate the breakthrough curves. This work focuses on simulating the effect of axial diffusion, solute concentration, ligand content, reaction kinetics, membrane thickness, and flow rate on the breakthrough behavior of the solute from an affinity membrane.

\section{Model Development}

The model system used for describing the affinity membrane separations is schematically represented in Fig. 1. The model is based on the isothermal adsorption of a single solute in dispersed plug flow through a porous affinity membrane onto which a ligand is immobilized. The bulk li-

\section{feed protein concentration, $C_{0}$}

flow velocity, $u_{i}$

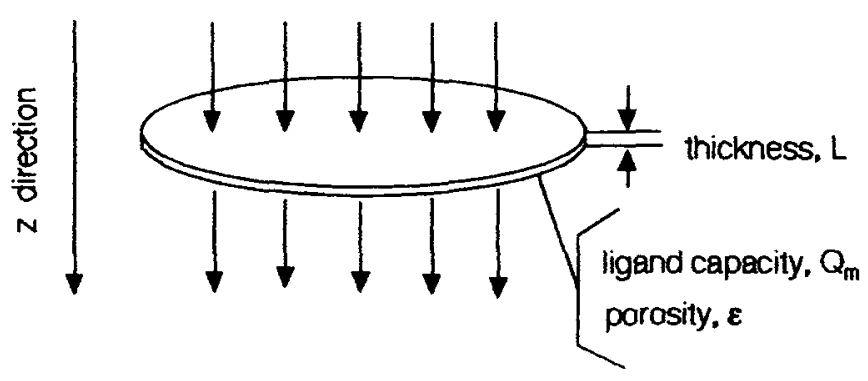

exit protein concentration, $C(L .1)$

Figure 1. Model of affinity membrane. 
quid has a solute concentration $c(z, t)$, with an interstitial velocity $u_{\mathrm{i}}$ through a membrane of thickness $L$, and void porosity $\varepsilon$. The total ligand capacity in the solid phase is $Q_{\mathrm{m}}$ and the immobilized ligand is distributed uniformly throughout the interior of the membrane. The concentration of the protein ligand complex in the solid phase is $q_{\mathrm{i}}(z, t)$.

Concentration gradients in radial direction are assumed to be negligible, because the time scale for boundary layer mass transfer is much less than that for axial convection [15]. One may replace the axial dispersion coefficient with the axial diffusion coefficient in the mathematical analysis of affinity membranes [9]. This change is also convenient because experimental measurements of molecular diffusion coefficients are readily available.

\subsection{Adsorption}

In the adsorption step, solute $\mathrm{P}$ of interest gets adsorbed onto the matrix on which the ligand $\mathrm{L}$ is immobilized. The affinity interaction between the protein and vacant immobilized ligand is assumed to be of the form: ${ }^{1}$

$$
\underset{\text { protein }}{\mathrm{P}}+\underset{\text { ligand }}{\mathrm{L}} \rightleftharpoons \underset{\text { complex }}{\mathrm{PL}}
$$

Monovalent adsorption is considered in the present study. Assuming that the affinity interactions is second order in forward direction and first order in reverse direction, the mass balance equation for solute adsorbed on solid phase can be written as:

$\frac{\partial q_{\mathrm{i}}}{\partial t}=k_{1} c\left(Q_{\mathrm{m}}-q_{\mathrm{i}}\right)-k_{2} q_{\mathrm{i}}$

Eq. (1) simplifies to Langmuir's isotherm at equilibrium.

$q_{\mathrm{i}}=\frac{Q_{\mathrm{m}} c}{\left(K_{\mathrm{d}}+c\right)}$

A mass balance over a section of the affinity membrane gives the following continuity relation:

$\frac{\partial c}{\partial t}+u_{\mathrm{i}} \frac{\partial c}{\partial z}+\frac{(1-\varepsilon)}{\varepsilon} \frac{\partial q_{\mathrm{i}}}{\partial t}=D_{\mathrm{i}} \frac{\partial^{2} c}{\partial z^{2}}$

The various terms in Eq. (3) account for variation of protein concentration with time, convective transfer of the protein, association rate, and axial diffusion, respectively.

Assuming that there is no protein in the membrane at the beginning, the initial conditions can be written as follows:

$$
\text { At } z \geq 0, \quad t=0: \quad \begin{aligned}
c & =0 \\
q_{\mathrm{i}} & =0
\end{aligned}
$$

1) List of symbols at the end of the paper.
In order to include axial diffusion at the front surface of the membrane and mixing at the exit of the membrane, the Danckwerts' [16] boundary conditions for frontal analysis are used.

$$
\text { At } z=0, \quad t>0: \quad u_{\mathrm{i}} c_{0}=u_{\mathrm{i}} c-D_{\mathrm{i}} \frac{\partial c}{\partial z}
$$

$$
\text { At } z=L, \quad t>0: \quad \frac{\partial c}{\partial z}=0
$$

In general, axial diffusion should be considered at the entrance of the membrane, because a steep first-order spatial gradient exists there. When axial diffusion is insignificant, i.e., $D_{\mathrm{i}}$ approaches zero, Eq. (6) reduces to $c=c_{0}$ at the entrance, which is the case of a step input.

\section{Numerical Solution Strategy}

The solution of the coupled non-linear PDEs together with the boundary conditions requires computation by numerical methods. Orthogonal collocation method [13, 14] is applied with respect to the space variable $z$ of the partial differential equations of the model to evaluate the breakthrough behavior of the solute. The mathematics of the orthogonal collocation method is given in the appendix.

The above equations are non-dimensionalized by the following dimensionless variables:

$y_{1}=q_{\mathrm{i}} / Q_{\mathrm{m}}, \quad y_{2}=c / c_{0}$

$z^{*}=z / L, \quad T=t u_{\mathrm{i}} / L$

In the new coordinates, the mass balance equations (1) to (3) get transformed to

$\frac{\partial y_{1}}{\partial T}=\mathrm{Da}_{\mathrm{a}} y_{2}\left(1-y_{1}\right)-\mathrm{Da}_{\mathrm{d}} y_{1}$

$y_{1}=\frac{y_{2}}{\left(H+y_{2}\right)}$

$\frac{\partial y_{2}}{\partial T}=\frac{1}{\operatorname{Pe}} \frac{\partial^{2} y_{2}}{\partial z^{* 2}}-\frac{\partial y_{2}}{\partial z^{*}}-\operatorname{Ld} \frac{\partial y_{1}}{\partial T}$

In terms of dimensionless variables, the initial and boundary conditions (Eq. (4) through (7) are as follows:

$y_{2}=0$

$y_{1}=0$

$1=y_{2}-\left(\frac{1}{\mathrm{Pe}}\right) \frac{\partial y_{2}}{\partial z^{*}}$

$\frac{\partial y_{2}}{\partial z^{*}}=0$ 
where the dimensionless numbers $\mathrm{Da}_{\mathrm{a}}, \mathrm{Da}_{\mathrm{d}}, H, \mathrm{Ld}, \mathrm{Pe}$ are defined as below:

$\mathrm{Da}_{\mathrm{a}}=\frac{k_{1} c_{0} L}{u_{\mathrm{i}}}, \quad \mathrm{Da}_{\mathrm{d}}=\frac{k_{2} L}{u_{\mathrm{i}}}, \quad H=\frac{K_{\mathrm{d}}}{c_{0}}$,

$\mathrm{Ld}=\frac{(1-\varepsilon)}{\varepsilon} \frac{Q_{\mathrm{m}}}{c_{0}}, \quad \mathrm{Pe}=\frac{L u_{\mathrm{i}}}{D_{\mathrm{i}}}$

The affinity membrane system is characterized by $\mathrm{Da}_{\mathrm{a}}$, $\mathrm{Da}_{\mathrm{d}}, H, \mathrm{Ld}, \mathrm{Pe}$, and defined by the system properties (axial diffusion coefficient of the solute $D_{\mathrm{i}}$ and ligand capacity $Q_{\mathrm{m}}$ ), membrane properties (porosity $\varepsilon$, thickness $L$ ), affinity kinetics (association rate constant $k_{1}$ and dissociation rate constant $k_{2}$ ), and the operational parameters (feed flow rate and feed solute concentration $c_{0}$ ). $\mathrm{Da}_{\mathrm{a}}$ is related to the efficiency of adsorption interaction and $\mathrm{Da}_{\mathrm{d}}$ represents the efficiency of desorption interaction. $H$ is a separation factor. Ld is a measure of the membrane capacity with respect to the feed solute concentration. Pe represents the axial dispersion resistance of the system.

The computer code for the governing mathematical equations is written in FORTRAN. The simulation is done by utilizing NAG routine (D02EAF) on Vax 88 system. This routine integrates a stiff system of first order ordinary differential equations, using a variable order variable step method implementing the backward differentiation formulae. Seven interior points in the membrane (total $=9$ ) are used for the simulation studies. The numerical code had been successfully implemented for similar mathematical model equations [17].

\section{Results and Discussion}

The effect of axial diffusion, solute concentration, ligand number, reaction kinetics, membrane thickness, and flow rate on the breakthrough curve has been evaluated for an affinity membrane. The solute concentration profiles are evaluated with respect to time, i.e., in form of a breakthrough curve. The base case parameter values used in the simulation [9] are listed in Tab. 1 and are used throughout unless stated otherwise. Breakthrough time is defined as the time required for the exit solute concentration to reach $20 \%$ to that of inlet solute concentration $\left(c=0.2 c_{0}\right)$.

Table 1. Data for simulation.

\begin{tabular}{ll}
\hline Parameter & Value \\
\hline$c_{0}[\mathrm{~mol} / 1]$ & $1.4 \cdot 10^{-6}$ \\
$\varepsilon[-1$ & 0.70 \\
$D_{\mathrm{i}}\left[\mathrm{cm}^{2} \mathrm{~s}^{-1}\right]$ & $1.0 \cdot 10^{-5}-6.7 \cdot 10^{-7}$ \\
$k_{1}\left[1 \mathrm{~mol}^{-1} \mathrm{~s}^{-1}\right]$ & $3.34 \cdot 10^{3}$ \\
$k_{2}\left[\mathrm{~s}^{-1}\right]$ & $4.68 \cdot 10^{-4}$ \\
$L[\mathrm{~cm}]$ & $1.5 \cdot 10^{-2}$ \\
$Q_{\mathrm{m}}[\mathrm{mol} / 1]$ & $1.58 \cdot 10^{-4}$ \\
$u_{\mathrm{i}}\left[\mathrm{cm} \mathrm{s}^{-1}\right]$ & $5.6 \cdot 10^{-5}$ \\
\hline
\end{tabular}

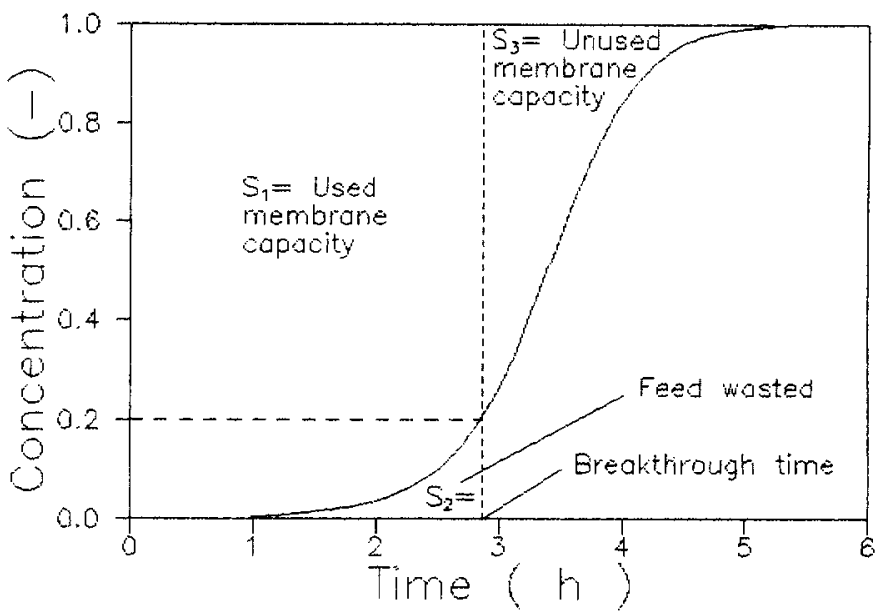

Figure 2. Breakthrough curve for affinity adsorption.

Fig. 2 shows a typical breakthrough curve for the adsorption step. Much of the information needed to evaluate membrane performance is contained in this curve. The shape of this curve is a result of a complex mix of equilibrium and non-equilibrium processes. The solute front takes $4.5 \mathrm{~min}$ (residence time) to pass through the membrane, and therefore, there is no fluid coming out before this time. The solution that comes out at $4.5 \mathrm{~min}$ contains unadsorbed solute at a concentration which depends on the capacity of the membrane. From 4.5 min onwards the effluent solute concentration increases with time. At very long times the membrane becomes saturated, and the effluent concentration equals the feed concentration. The maximum capacity of the membrane for a given inlet concentration is equal to the area behind the breakthrough curve. The amount of solute that remains in the effluent is, of course, the area under the curve. For quantitative comparison purpose, the following design parameters are defined: Solute recovery efficiency [18], ligand utilization efficiency [18], and thickness of unused membrane (TUM) are defined in terms of area $S_{1}$ (used membrane capacity), $S_{2}$ (feed wasted), and $S_{3}$ (unused membrane capacity) as shown in Fig. 2.

1. Solute recovery efficiency $=\frac{S_{1}}{S_{1}+S_{2}}$

2. Ligand utilization efficiency $=\frac{S_{1}}{S_{1}+S_{3}}$

3. Thickness of unused membrane, TUM $=\frac{S_{3}}{S_{1}+S_{3}} L$

4. The mass transfer zone (MTZ) is the region where concentration is changing and thus mass transfer is occurring. The width of MTZ in time units is easily measured from the breakthrough curve. Usually, MTZ is arbitrarily measured from a concentration of $0.05 c_{0}$ to $0.95 c_{0}$.

The determination of the breakthrough point and the shape of the curve affects the efficiencies of adsorption. Solute recovery and membrane utilization efficiencies are calculated at a breakthrough concentration of $c=0.2 c_{0}$ for various parameters. Tab. 2 presents the design parameters. 
Table 2. Solute recovery efficiency, ligand utilization efficiency, TUM, and MTZ for various parameter values.

\begin{tabular}{lllll}
\hline Parameter & $\begin{array}{l}\text { Solute recovery } \\
\text { efficiency }[\%]\end{array}$ & $\begin{array}{l}\text { Ligand utilization } \\
\text { efficiency }[\%]\end{array}$ & $\begin{array}{l}\text { TUM } \\
{[\mathrm{cm}]}\end{array}$ & $\begin{array}{l}\text { MTZ } \\
{[\mathrm{h}]}\end{array}$ \\
\hline $\mathrm{Pe}$ & & & & \\
0.025 & 91.9 & 70.0 & $4.5 \cdot 10^{-3}$ & 14.4 \\
1.25 & 96.0 & 83.0 & $2.55 \cdot 10^{-3}$ & 2.3 \\
$c_{0}$ & & & & \\
$7.0 \cdot 10^{-7}$ & 96.5 & 74.0 & $3.9 \cdot 10^{-3}$ & 6.0 \\
$1.4 \cdot 10^{-6}$ & 96.0 & 83.0 & $2.55 \cdot 10^{-3}$ & 2.3 \\
$\mathrm{Ld}$ & & & & \\
48.4 & 96.0 & 83.0 & $2.55 \cdot 10^{-3}$ & 2.3 \\
107 & 97.8 & 85.0 & $2.25 \cdot 10^{-3}$ & 4.3 \\
$\mathrm{Da}_{\mathrm{a}}$ & & & & \\
0.56 & 93.6 & 67.5 & $4.87 \cdot 10^{-3}$ & 3.6 \\
1.25 & 96.0 & 83.0 & $2.55 \cdot 10^{-3}$ & 2.3 \\
$L$ & & & & \\
$1.5 \cdot 10^{-2}$ & 96.0 & 83.0 & $2.55 \cdot 10^{-3}$ & 2.3 \\
$3.0 \cdot 10^{-2}$ & 98.0 & 90.0 & $1.5 \cdot 10^{-3}$ & 2.9 \\
$u_{\mathrm{i}}$ & & & & \\
$1 \cdot 10^{-4}$ & 95.0 & 72.0 & $4.2 \cdot 10^{-3}$ & 1.0 \\
$5.6 \cdot 10^{-5}$ & 96.0 & 83.0 & $2.55 \cdot 10^{-3}$ & 2.3 \\
\hline
\end{tabular}

Fig. 3 explains the effect of axial diffusion on breakthrough curve. Axial diffusion affects the sharpness of the breakthrough curve. At a small Péclet number $\left(\mathrm{Pe}=L u_{\mathrm{i}} / E_{\mathrm{z}}\right)$, the breakthrough curves are broadened, the time of total saturation is delayed, and the loading capacity at the point of breakthrough is decreased. As Pe increases, the breakthrough curves become sharper. The higher the Péclet number, the lesser the axial diffusion, and hence the lesser the back mixing. This membrane operates with a Péclet number of 1.25. Pe is small when the membrane is thin, the association rate is low, and axial diffusion coefficient is large. The breakthrough curve for Péclet number 0.025 has lower solute recovery and adsorbent utilization efficiencies, higher TUM and MTZ compared to that of the base case curve. Higher Péclet numbers are preferred for an efficient operation.

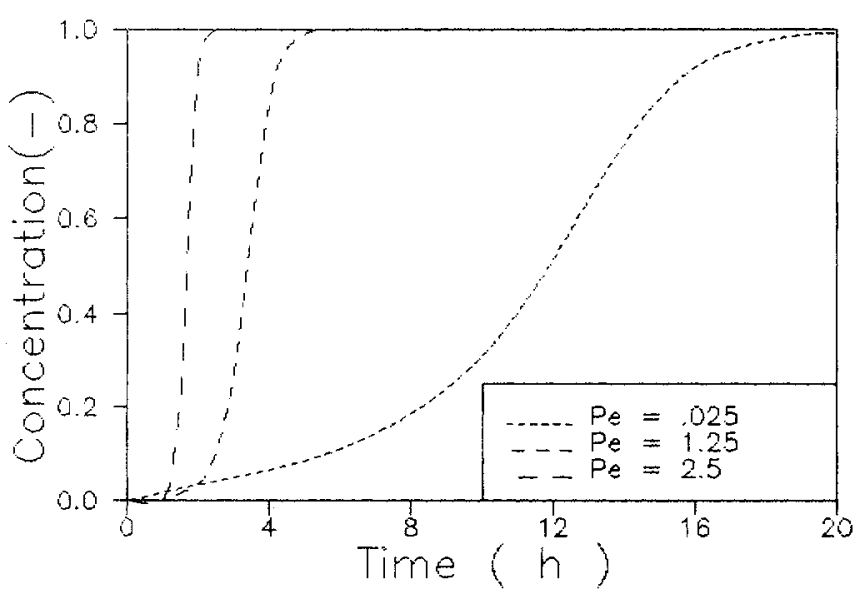

Figure 3. Effect of Péclet number on breakthrough curve.
Fig. 4 displays the effect of solute concentration on breakthrough curve. The change in inlet solute concentration markedly affects the shape and position of the breakthrough curve. The higher the solute concentration, the faster the breakthrough. The results in Tab. 2 show that solute recovery efficiency decreases and adsorbent utilization efficiency increases as $c_{0}$ increases. Lean feed is found to be better for solute recovery. However, the quantity of solution to be supplied is large when the solute concentration is low and an optimum value has to be arrived at. Lean feed results in an increase of both TUM and MTZ.

Fig. 5 depicts the effect of Ligand number on breakthrough curve. For the same inlet solute concentration, breakthrough curves are evaluated for three different Ligand numbers. Ligand number is a measure of characteristic ligand capacity of the membrane to characteristic solute in feed solution. The higher ligand capacity leads to more uptake of solute and, therefore, better bed utilization. It can be observed from Tab. 2 that MTZ is proportionate to the increase in Ld.

Fig. 6 evaluates the effect of Damköhler number (adsorption) on the breakthrough behavior of the solute. $\mathrm{Da}_{\mathrm{a}}$ is a

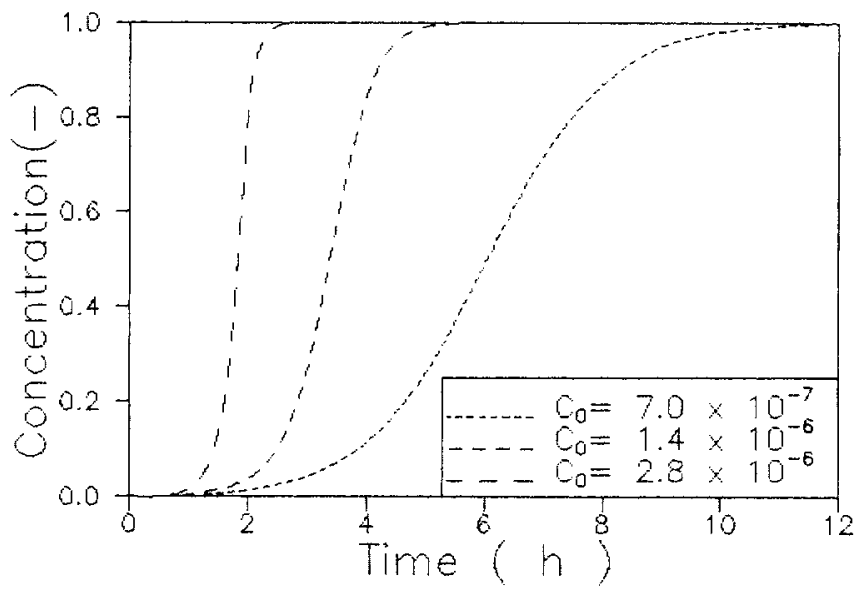

Figure 4. Effect of solute concentration on breakthrough curve.

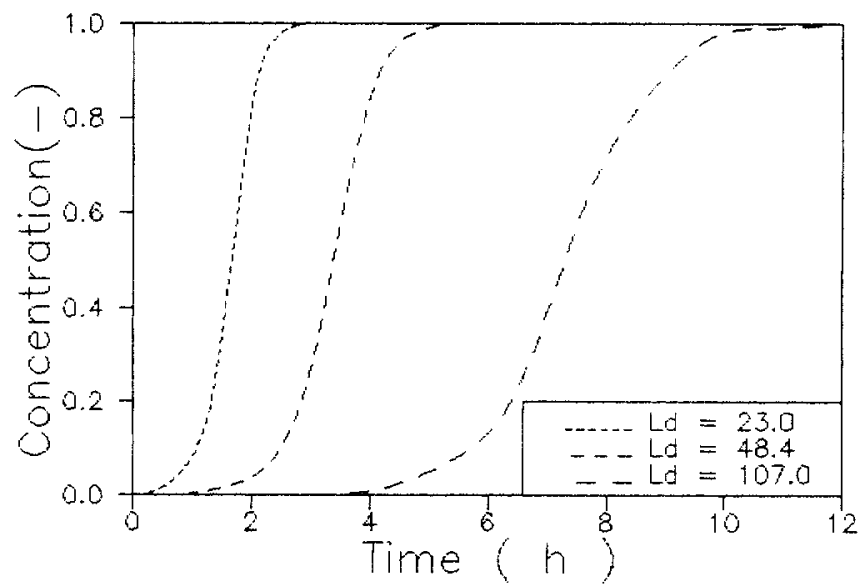

Figure 5. Effect of Ligand number on breakthrough curve. 


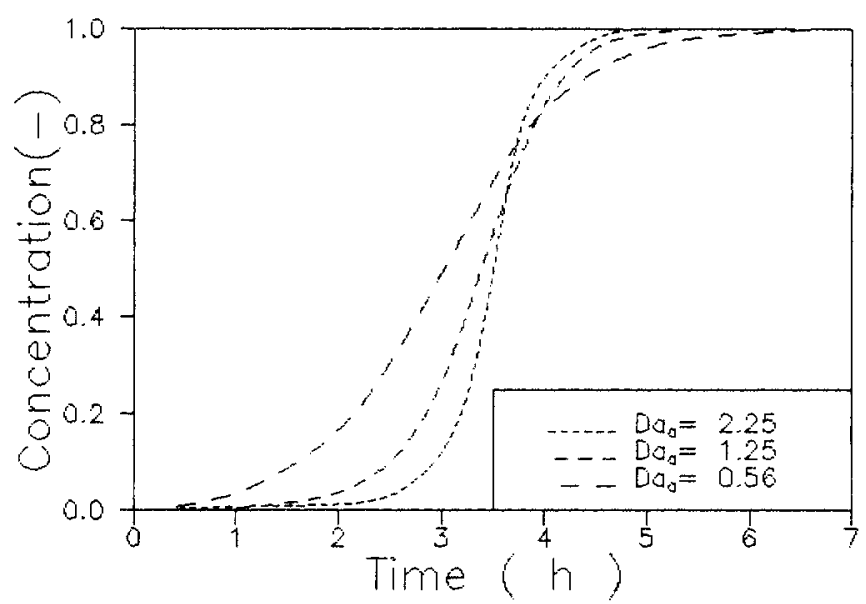

Figure 6. Effect of Damköhler number on breakthrough curve.

ratio of residence time to characteristic adsorption time. Higher $\mathrm{Da}_{\mathrm{a}}$ 's imply fast adsorption. The larger the $\mathrm{Da}_{\mathrm{a}}$, the sharper the breakthrough, thus improving adsorption efficiency. Faster rates of the forward rate constant allow local equilibrium conditions to be approached more quickly. Kinetic rate constants can be changed by changing the $\mathrm{pH}$ of the medium. Higher $\mathrm{Da}_{\mathrm{a}}$ reduces both TUM and MTZ.

Fig. 7 examines the effect of membrane thickness on the breakthrough curve. Higher thickness implies higher capacity, which leads to better solute recovery and bed utilization efficiencies. TUM decreases as membrane thickness increases. It can be observed from Tab. 2 that MTZ is proportional to the increase in membrane thickness.

Fig. 8 shows the effect of flow rate on the breakthrough behavior of the protein. Breakthrough is faster for higher flow rates. When the flow rate is low, a decrease in the spreading of the curve results in large solute recovery and bed utilization efficiencies. High flow rates result in poor adsorption efficiencies. Low flow rates increase the time that the solute is in contact with the solid phase, allowing more time for adsorption and permitting near-local

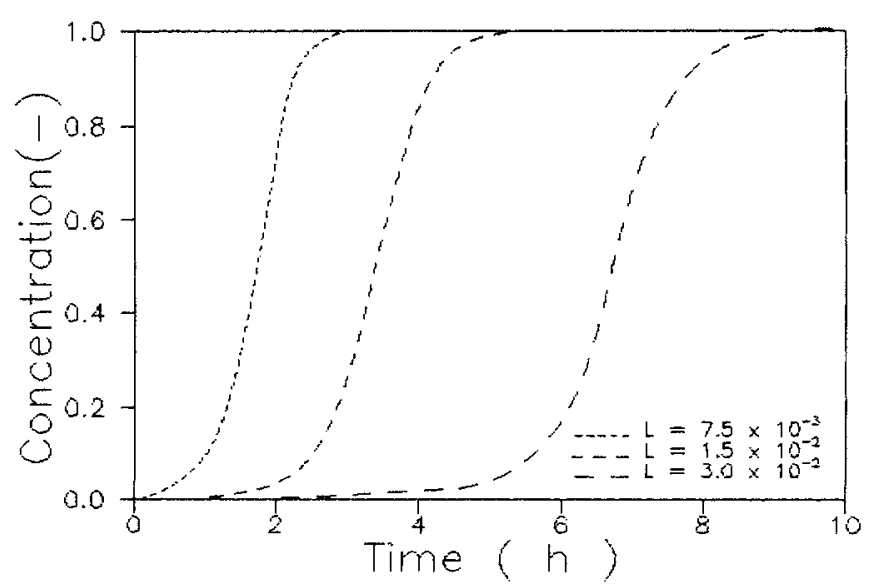

Figure 7. Effect of membrane thickness on breakthrough curve.

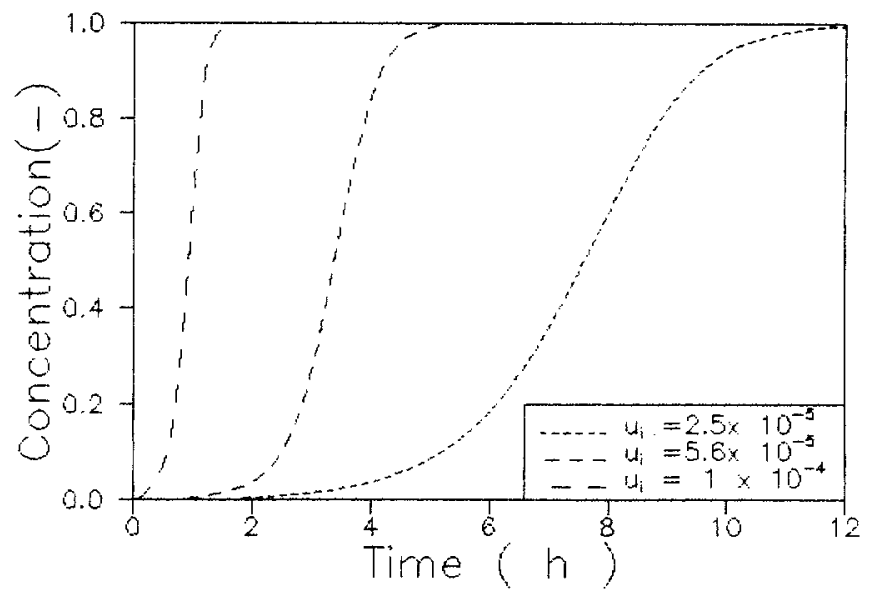

Figure 8. Effect of flow rate on breakthrough curve.

equilibrium conditions. High flow rates result in high TUM and low MTZ.

\section{Conclusions}

Affinity purification is based on specific biological recognition and selective binding described by a non-linear rate expression. In the present study the following parameters have been shown to affect the breakthrough behavior of an affinity membrane: Péclet number Pe, inlet solute concentration, Ligand number Ld, Damköhler number $\mathrm{Da}_{\mathrm{a}}$, membrane thickness, and flow rate. The effect of axial diffusion is significant and affects solute recovery and adsorbent utilization efficiencies, TUM and MTZ. For thin membranes, the association kinetics may limit the flow velocity to such an extent that axial diffusion becomes a dominant and undesirable mechanism for axial transport of protein through the membrane. In order to completely eliminate axial diffusion, it is necessary to operate the present affinity membrane system at a condition of $\mathrm{Pe}>2.5$. A practical consequence of these results is that thicker membranes in the form of stacks of membranes should be a better design than single thin affinity membranes. A thorough ex-

Table 3. Collocation points for simulation.

\begin{tabular}{lll}
\hline Number & $\alpha=0, \beta=0$ & $\alpha=2, \beta=1$ \\
\hline 1 & 0.0 & 0.0 \\
2 & 0.02544604 & \\
& 38 & 0.0465319745 \\
3 & 0.12923440 & \\
& 72 & 0.1457947205 \\
4 & 0.29707742 & \\
& 43 & 0.2859798043 \\
5 & 0.5 & 0.4538708651 \\
6 & 0.70292257 & \\
& 57 & 0.6310610011 \\
7 & 0.87076559 & \\
8 & 28 & 0.7949622505 \\
& 0.97455395 & 0.9230493840 \\
9 & 62 & 1.0 \\
\hline
\end{tabular}


perimental verification of the model equations presented in this work remains to be done. By using the same governing mathematical equations, but by changing the initial and boundary conditions, washing and elution steps can be modeled. Design strategies have been obtained through solute recovery efficiency, ligand utilization efficiency, thickness of unused membrane, and width of mass transfer zone.

\section{Appendix}

The choice of collocation points is important especially if low order approximations are to be used. Villadsen and Michelsen [14] suggested in general the use of the roots of Jacobi polynomials which have the following orthogonal property:

$\int_{0}^{1} x^{\beta}(1-x)^{\alpha} P_{\mathrm{J}}(x) P_{\mathrm{N}}(x) \mathrm{d} x=0$

The choice of $\alpha=0$ and $\beta=0$ yields the standard Gaussian quadrature points in $(0,1)$ as the collocation points. However, as the absolute value of the solute concentration gradient is maximum at $x=0$, it is advantageous to shift the collocation points closer to the inlet. This can be done by choosing $\alpha>\beta$. A choice of $\alpha=2$ and $\beta=1$ shifts the collocation points closer to the inlet without deemphasizing too much near the outlet. Hence, reasonable accuracy is obtained for the entire width of the process zone. Collocation points for two different sets of $\alpha, \beta$ are given in Tab. 3 .

A possible choice of trial function of the asymmetric case is

$y(x)=x+x(1-x) \sum_{i=1}^{N} a_{\mathrm{i}} P_{\mathrm{i}-1}(x)$

For simplicity in deriving the derivative matrices, the above series can be written as:

$y(x)=\sum_{i=1}^{N+2} d_{\mathrm{i}} x^{\mathrm{i}-1}$

i.e.,

$y\left(x_{\mathrm{j}}\right)=d_{1}+d_{2} x_{\mathrm{j}}+d_{3} x_{\mathrm{j}}^{2}+\ldots d_{\mathrm{N}} x_{\mathrm{j}}^{\mathrm{N}-1}+d_{\mathrm{N}+1} x_{\mathrm{j}}^{\mathrm{N}}+d_{\mathrm{N}+2} x_{\mathrm{j}}^{\mathrm{N}+}$

First derivative and Laplacian of this expression can be taken and evaluated at the collocation points:

$$
\begin{aligned}
& y\left(x_{\mathrm{j}}\right)=\sum_{i=1}^{N+2} d_{\mathrm{i}} x_{\mathrm{j}}^{\mathrm{i}-1} \\
& \left.\frac{\mathrm{d} y(x)}{\mathrm{d} x}\right|_{x_{\mathrm{j}}}=\left.\sum_{i=1}^{N+2} \frac{\mathrm{d} x^{\mathrm{i}-1}}{\mathrm{~d} x}\right|_{x_{\mathrm{j}}} \mathrm{d}_{\mathrm{i}}
\end{aligned}
$$

$$
\left.\nabla^{2} y\right|_{x_{\mathrm{j}}}=\left.\sum_{i=1}^{N+2} \nabla^{2}\left(x^{\mathrm{i}-1}\right)\right|_{x_{\mathrm{j}}} \mathrm{d}_{\mathrm{i}}
$$

These can be written in matrix notation as follows. The square matrices have $(\mathrm{N}+2) \times(\mathrm{N}+2)$ elements:

$y=Q d, \quad \frac{\mathrm{d} y}{\mathrm{~d} x}=c d, \quad \nabla^{2} y=D d$

$Q_{\mathrm{ji}}=x_{\mathrm{j}}^{\mathrm{i}-1}, \quad c_{\mathrm{ji}}=(i-1) x_{\mathrm{j}}^{\mathrm{i}-2}, \quad D_{\mathrm{ji}}=(i-1)(i-2)\left(x_{\mathrm{j}}^{\mathrm{i}-3}\right)$

Solving for $d$ gives:

$\frac{\mathrm{d} y}{\mathrm{~d} x}=c Q^{-1} y=A y, \quad \nabla^{2} y=D Q^{-1} y=B y$

Thus, the derivative at any collocation point is expressed in terms of the value of the trial function at the collocation points.

The following quadrature formula is used for the accurate evaluation of the integrals:

$\int_{0}^{1} f(x) \mathrm{d} x=\sum_{i=1}^{N+2} W_{\mathrm{j}} f\left(x_{\mathrm{j}}\right)$

To determine $W_{\mathrm{j}}$, we evaluate the above equation for $f_{\mathrm{j}}=x^{\mathrm{i}-1}$ :

$$
\begin{aligned}
& \int_{0}^{1} x^{\mathrm{i}-1} \mathrm{~d} x=\sum_{i=1}^{N+2} x_{\mathrm{j}}^{\mathrm{i}-1} W_{\mathrm{j}}=\frac{1}{i} \\
& W Q=f \quad W=f Q^{-1}
\end{aligned}
$$

In the case of partial differential equations, the partial derivatives become ordinary derivatives at each collocation point as one independent variable is fixed.

Application of the orthogonal collocation method to the dimensionless model equations [Eqs. (8) through (14)] lead to the following set of working equations:

$\frac{\mathrm{d} Y_{1 \mathrm{j}}}{\mathrm{d} T}=\mathrm{Da}_{\mathrm{a}} Y_{2 \mathrm{j}}\left(1-Y_{1 \mathrm{j}}\right)-\mathrm{Da}_{\mathrm{d}} Y_{1 \mathrm{j}}$

$Y_{1 \mathrm{j}}=\frac{Y_{2 \mathrm{j}}}{\left(H+Y_{2 \mathrm{j}}\right)}$

$\frac{\mathrm{d} Y_{2 \mathrm{j}}}{\mathrm{d} T}=\frac{1}{\operatorname{Pe}} \sum_{i=1}^{N+2} B_{\mathrm{ji}} Y_{2 \mathrm{i}}-\sum_{i=1}^{N+2} A_{\mathrm{ji}} Y_{2 \mathrm{i}}-\mathrm{Ld} \frac{\mathrm{d} Y_{1 \mathrm{j}}}{\mathrm{d} T}$

$Y_{2 j}=0$

$Y_{1 \mathrm{j}}=0$ 
$1=Y_{21}-\frac{1}{\operatorname{Pe}} \sum_{i=1}^{N+2} A_{1, \mathrm{i}}^{\prime} Y_{2 \mathrm{i}}$

$\sum_{i=1}^{N+2} A_{\mathrm{N}+2, \mathrm{i}}^{\prime} Y_{2 \mathrm{i}}=0$

where $j=2$ to $N+1$.

These equations are integrated numerically using the NAG routine (D02EAF) on Vax 88 system.

\section{Acknowledgement}

The author would like to thank CSIR for the financial assistance (RA no. 9/79 (473)/94-EMR.I, dated July 14, 1994).

Received: July 24, 1995 [CET 763]

\section{Symbols used}

\begin{tabular}{|c|c|c|}
\hline$c$ & {$[\mathrm{~mol} / \mathrm{l}]$} & $\begin{array}{l}\text { solute concentration at any axial position in the } \\
\text { fluid phase }\end{array}$ \\
\hline$c_{0}$ & {$[\mathrm{~mol} / \mathrm{l}]$} & feed concentration in the fluid phase \\
\hline $\mathrm{Da}_{\mathrm{a}}$ & {$[-]$} & Damköhler number for adsorption \\
\hline $\mathrm{Da}_{\mathrm{d}}$ & {$[-]$} & Damköhler number for desorption \\
\hline$D_{\mathrm{i}}$ & {$\left[\mathrm{cm}^{2} \mathrm{~s}^{-1}\right]$} & axial diffusion coefficient \\
\hline$H$ & {$[-]$} & dimensionless separation factor \\
\hline$K_{\mathrm{d}}$ & {$[\mathrm{mol} / \mathrm{l}]$} & dissociation constant, $=k_{2} / k_{1}$ \\
\hline$k_{1}$ & {$\left[1 \mathrm{~mol}^{-1} \mathrm{~s}^{-1}\right]$} & second order rate constant \\
\hline$k_{2}$ & {$\left[\mathrm{~s}^{-1}\right]$} & first order rate constant \\
\hline$L$ & {$[\mathrm{~cm}]$} & membrane thickness \\
\hline Ld & {$[-]$} & ligand number \\
\hline $\mathrm{Pe}$ & {$[-]$} & Péclet number, $=L u_{\mathrm{i}} / D_{\mathrm{i}}$ \\
\hline$q_{\mathrm{i}}$ & {$[\mathrm{mol} / 1]$} & $\begin{array}{l}\text { local adsorbed solute concentration in the solid } \\
\text { phase }\end{array}$ \\
\hline$Q_{\mathrm{m}}$ & [1] & $\begin{array}{l}\text { maximum ligand concentration in the solid } \\
\text { phase based on the solid volume }\end{array}$ \\
\hline$t$ & [s] & time \\
\hline$T$ & {$[-]$} & dimensionless time, $=t u_{\mathrm{i}} / L$ \\
\hline$u_{\mathrm{i}}$ & {$\left[\mathrm{cm} \mathrm{s}^{-1}\right]$} & interstitial velocity \\
\hline
\end{tabular}

$\begin{array}{lll}y_{1} & {[-]} & \begin{array}{l}\text { dimensionless local adsorbed solute concentra- } \\ \text { tion in the solid phase, }=q_{\mathrm{i}} / Q_{\mathrm{m}} \\ \text { dimensionless solute concentration at any axial } \\ \text { position in the fluid phase, }=c / c_{0}\end{array} \\ y_{2} & {[-]} & \begin{array}{l}\text { axial position in the membrane } \\ \text { dimensionless axial coordinate }\end{array}\end{array}$

Greek symbols

$\varepsilon \quad[-] \quad$ membrane void porosity

\section{References}

[1] Pungor, E., Afeyan, N.B., Gordon, N.F., Cooney, C.L., Bio/Technology 5 (1987) pp. 604.

[2] Huang, S.H., Roy, S., Hou, K.C., Tsao, T.C., Biotechnol. Prog. 4 (1984) pp. 159.

[3] Luong, J.H.T., Nguygen, A.L., Male, K. B., Bio/Technology 5 (1987) pp. 564.

[4] Burns, M.A., Graves, D. J., Biotechnol. Prog. I (1985) pp. 95.

[5] Chase, H.A., Draeger, N. M., J. Chromatogr. 129 (1992) pp. 597.

[6] Afeyan, N.B., Fulton, S.P., Gordon, N.F., Mazsaroff, I., Varady, L., Regnier, F.E., Bio/Technology 8 (1990) pp. 203.

[7] Zale, S.E., Kessler, S.B., Goffe, R.A., Matson, S. L., North American Membrane Society Second Annual National Meeting, Syracuse, NY, Jan., 1988.

[8] Brandt, S., Goffe, R.A., Kessler, S.B., O'Connor, J.L., Zale, S. E., Bio/Technology 6 (1988) pp. 779.

[9] Suen, S. Y., Etzel, M.R., Chem. Eng. Sci. 47 (1992) pp. 1355.

[10] Arnold, F.H., Blanch, H.W., Chem. Eng. J. Part B 30 (1985) pp. 9.

[11] Blankstein, L.A., Dohrman, L., Am. Clin. Prod. Rev. 11 (1985) pp. 33.

[12] Chase, H.A., J. Chromatogr. 179 (1984) pp. 179-202.

[13] Finlayson, B.A., in: Non-linear Analysis in Chemical Engineering, McGraw-Hill, New York 1980.

[14] Villadsen, J., Michelsen, M.L., in: Solution of Differential Equation Models by Polynomial Approximation, Prentice-Hall, New Jersey 1978.

[15] Athalye, A. M., Gibbs, S. J., Lightfoot, E. N., J. Chromatogr. 71 (1991) pp. 71-85.

[16] Danckwerts, P.V., Chem. Eng. Sci. 2 (1953) pp. 1.

[17] Sridhar, P., Sastri, N.V.S., Modak, J.M., Mukherjee, A. K., Chem. Eng. Technol. 17 (1994) pp. $422-429$.

[18] Bo-Lun, Y., Montonobu, G., Shigeo, G., Colloids Surf. 37 (1989) pp. 369. 